\title{
Developing the Automatic Water Surface Pollution Search and Identification Method Using Satellite Optical Imagery
}

\author{
Victor V. Zamshin, Vitaly D. Kharchenko, Vasilisa N. Khodaeva \\ AEROCOSMOS Research Institute for Aerospace Monitoring, Moscow, Russia \\ office@aerocosmos.info
}

\begin{abstract}
Here we describe the results from development and testing of the automatic water surface pollution search and identification method using satellite optical imagery. A search of water surface characterized by the specific shape of spectral reflectance curve distinguishing from typical background curve in visible and near-IR bands was carried out within the test area (Russian Black Sea areas) using satellite data (Landsat-7/8 and Sentinel-2A/B, March 2017 - August 2018). When such sites were detected, additional research was carried out, including the difference between the anomaly and background water spectra, spatial shape, size, relation of this area with potential pollution sources etc. The results of this research allowed us to make a conclusion on the origin of the registered anomalies. Possible thematic application of the proposed method by the example of water surface oil pollution search and identification are described here.
\end{abstract}

Keywords: remote sensing, satellite optical imagery, pollutions, marine surface, automatic analysis, GIS, monitoring

Copyright (C2019 by the paper's authors. Copying permitted for private and academic purposes 


\title{
РАЗРАБОТКА АВТОМАТИЗИРОВАННОГО МЕТОДА ПОИСКА И ИДЕНТИФИКАЦИИ ЗАГРЯЗНЕНИЙ ВОДНОЙ ПОВЕРХНОСТИ ПО КОСМИЧЕСКИМ ОПТИЧЕСКИМ ИЗОБРАЖЕНИЯМ
}

\author{
В.В. Замшин, В.Д. Харченко, В.Н. Ходаева \\ Научно-исследовательский институт аэрокосмического мониторинга "АЭРОКОСМОС", Москва, \\ Россия \\ office@aerocosmos.info
}

Приводятся результаты разработки и экспериментальной апробации автоматизированного метода поиска и идентификации загрязнений водной поверхности по космическим оптическим изображениям. По данным космических съёмок (спутники Landsat-7/8, Sentinel-2A/B, период исследования - с марта 2017 года по август 2018 года) в пределах тестового района (российский сектор Чёрного моря) выполнялся поиск участков водной поверхности, характеризующихся особой формой кривой спектральной отражательной способности, отличающейся от типичной фоновой кривой в видимом и ближнем инфракрасном диапазонах спектра. При обнаружении таких участков выполнялось их дополнительное исследование, в том числе определялся характер отличия спектра аномалии от спектра фоновой водной поверхности, пространственная форма, размеры, приуроченность участка к потенциальным источникам загрязнений и др., на основании чего делался вывод о происхождении регистрируемых аномалий. Показаны возможности тематического применения предложенного метода на примере поиска и идентификации нефтяных загрязнений водной поверхности.

Ключевые слова: дистанционное зондирование, космическая оптическая съёмка, загрязнения, морская поверхность, автоматизированный анализ, геоинформационные системы, мониторинг.

\section{Введение}

В морскую среду постоянно поступают загрязняющие вещества, как естественного, так и антропогенного происхождения, в том числе углеводороды, сточные воды и др. В связи с этим необходимо проводить мониторинг морских акваторий и, прежде всего, их прибрежных участков, характеризующихся интенсивной хозяйственной деятельностью. Для решения задач мониторинга морской среды и получения данных о различного типа загрязнениях акваторий целесообразно применять данные дистанционного зондирования Земли, в том числе космические оптические изображения [1-5]. При решении задач мониторинга морских акваторий основными преимуществами космических оптических изображений перед другими типами данных являются: возможность использования различных спектральных диапазонов, возможность вести работу на труднодоступных участках, возможность одновременного охвата больших площадей, регулярность наблюдений, стандартизованность получаемой информации и др. [3-11].

Актуальными задачами для организации эффективного мониторинга загрязнений водной поверхности с использованием космических оптических изображений являются: освоение большого массива оперативной и архивной информации, представленной главным образом многоспектральными изображениями среднего и высокого пространственного разрешения; снижение временных затрат на обработку; повышение точности и объективности методов поиска 
и идентификации загрязнений. В этой связи необходимо совершенствовать существующие и разрабатывать новые методы обработки космических оптических изображений водной поверхности, отвечающие современным вызовам в области дистанционного мониторинга загрязнений акваторий $[3,4,7,12]$.

\section{Описание метода}

В исследовании предложен автоматизированный метод поиска и идентификации загрязнений водной поверхности по космическим оптическим панхроматическим и многоспектральным изображениям, блок-схема которого приведена на рис.1.
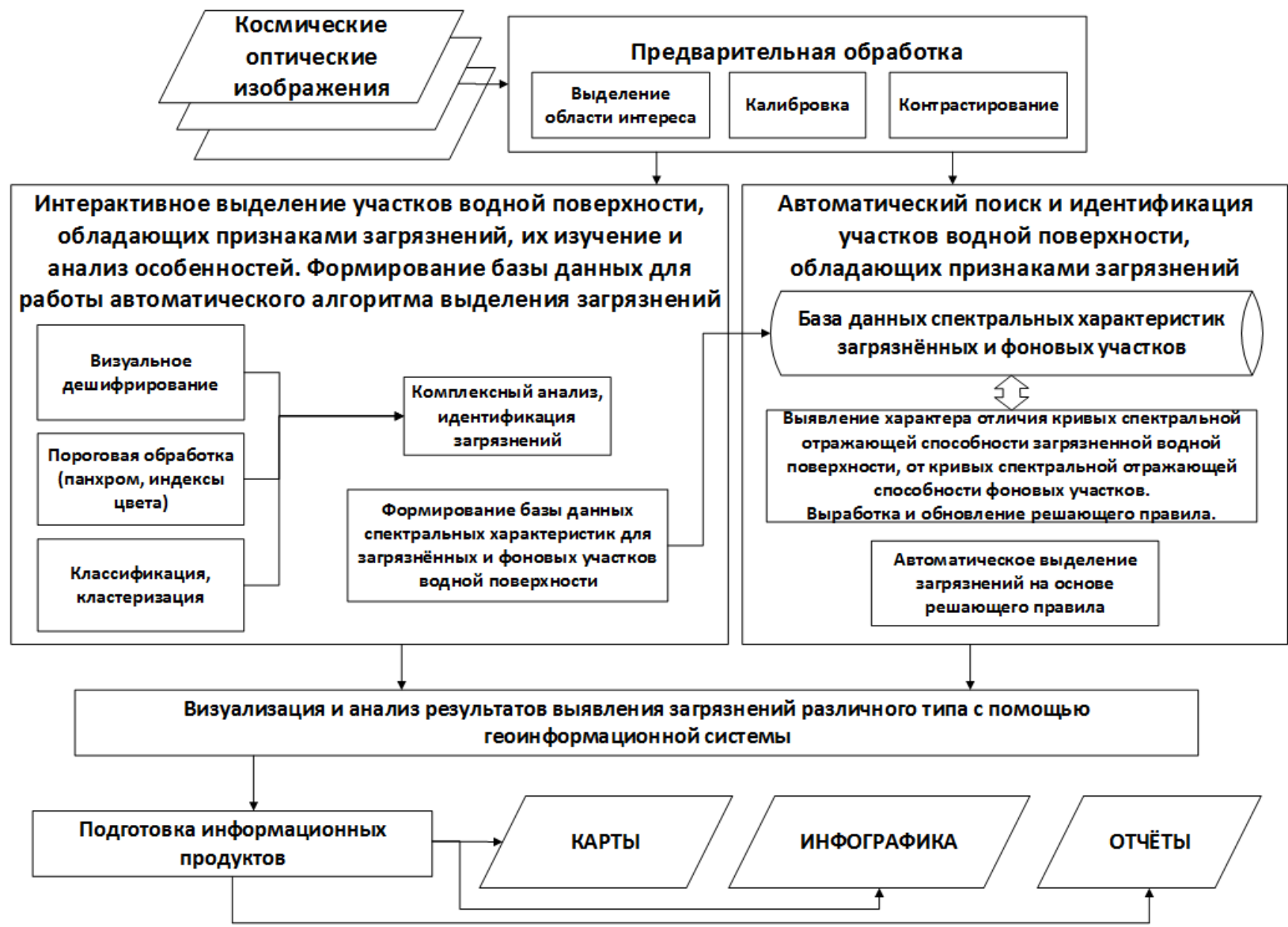

Рис. 1. Блок-схема автоматизированного метода поиска и идентификации загрязнений водной поверхности по космическим оптическим изображениям

Как видно из рис.1, после выполнения предварительной обработки (выделение области интереса по географическим координатам, радиометрическая калибровка с получением величин спектральной отражательной способности водной поверхности, формирование визуализаций с различными комбинациями каналов и настройками контраста) могут применяться 2 различных способа тематической обработки (выделения и идентификации загрязнений).

Первый способ предполагает интерактивное (с участием оператора-дешифровщика) выделение зон, обладающих признаками загрязнений (например, нефтепроявлений или глубинных стоков). Для этого оператором-дешифровщиком применяются такие приёмы как визуальное дешифрирование, пороговая обработка панхроматического канала или вычисленного индекса цвета [4,13-15], а также кластеризация и контролируемая классификация (см. рис.1,2,3). В большинстве случаев на основе комбинирования этих приёмов можно достаточно уверенно 
обнаружить, определить границы и предварительно идентифицировать загрязнения. Такой способ характеризуется невысокой степенью автоматизации, однако он позволяет сформировать базу данных спектральных характеристик загрязнённых и фоновых участков водной поверхности (БДСХ), которая впоследствии может быть использована для автоматического поиска и идентификации загрязнений.

При достаточном уровне наполнения БДСХ (достичь такого уровня планируется в рамках продолжения настоящего исследования) в действие может быть введён второй способ тематической обработки космических оптических изображений водной поверхности, в рамках которого поиск и идентификация загрязнений будет осуществляться автоматически (см. рис.1). Для этого требуется проанализировать массив данных, записанных в БДСХ, и выявить стабильные признаки в характере отличия кривых спектральной отражающей способности загрязненной водной поверхности от кривых спектральной отражающей способности фоновых участков. После этого производится выработка решающих правил, позволяющих автоматически выделять и идентифицировать загрязнения различных типов, при этом важно предусмотреть возможность обновления настроечных параметров обработки при поступлении в БДСХ новых верифицированных данных.
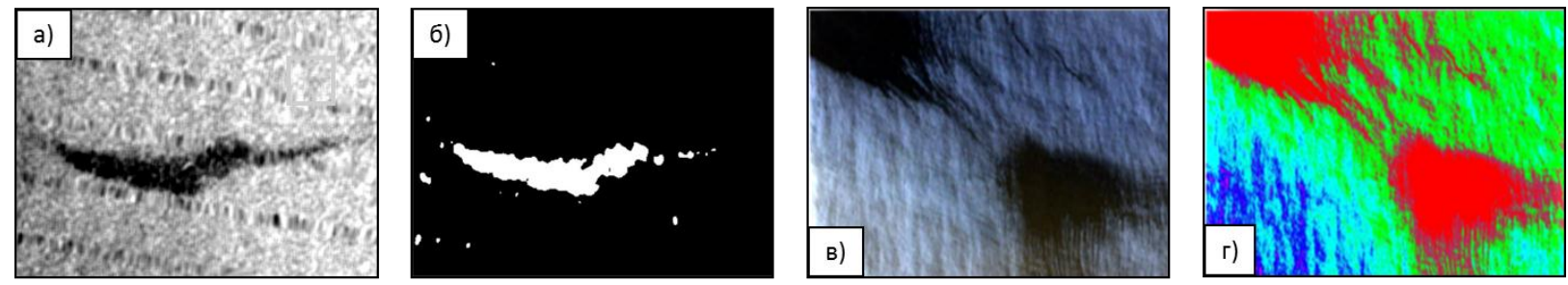

Рис. 2. Примеры пороговой обработки панхроматического а),б) и кластеризации многоспектрального в),г) космических оптических изображений для автоматизации выделения загрязнений водной поверхности: а)

- фрагмент панхроматического изображения (Landsat-7); б) - маска нефтяного слика, полученная в результате пороговой обработки (а); в) - фрагмент космического многоспектрального изображения (Landsat-8); г) - результат кластеризации (в) с применением алгоритма ISODATA (красный кластер соответствует нефтяному загрязнению)


Рис. 3. Примеры контролируемой классификации а),б) и пороговой обработки в),г) космического оптического многоспектрального изображения (Landsat-8) для автоматизации выделения загрязнений водной поверхности по: a) - фрагмент изображения для района воздействия глубинных стоков (синтез в натуральных цветах); б) - выделение плюма глубинных стоков на (а) с помощью классификации с обучением (розовый класс соответствует загрязнению); в) - фрагмент поля распределения индекса цвета, вычисленного как отношение синего и зелёного каналов (а); г) - маска загрязнений, вычисленная на основании пороговой обработки (в)

Несмотря на значительную вариабельность оптических аномалий, связанных с различными типами загрязнений акваторий, регистрируемых при космической оптической съёмке, принципиальная возможность автоматизации процесса поиска и идентификации таких аномалий с приемлемой точностью существует. Например, в работах $[13,15]$ показана типичность и относительная стабильность свойств оптических аномалий, обусловленных всплытием на морскую поверхность сточных вод. В настоящей работе (см. далее) показана относительная стабильность свойств оптических аномалий, обусловленных нефтезагрязнениями 
для случая, когда нефть визуализируется на многоспектральных изображениях в виде пятен с отрицательными контрастами.

В рамках реализации автоматической ветви метода поиска и идентификации загрязнений водной поверхности по космическим оптическим изображениям (см. рис.1) на данном этапе исследований на основании анализа информации, сохранённой в БДСХ, ведётся разработка специализированных индексов цвета и наборов пороговых значений для них, ориентированных на выявление конкретных типов загрязнений водной поверхности. При накоплении в БДСХ значительного объёма информации, в качестве средств, обеспечивающих поиск и идентификацию загрязнений, планируется задействовать нейросетевые решения.

\section{Пример использования метода}

Апробация предложенного метода поиска и идентификации загрязнений водной поверхности проводилась на акватории российского сектора Чёрного моря вблизи полуострова Крым. На этом участке с марта 2017 года по август 2018 года проводился космический мониторинг нефтяных загрязнений по оптическим изображениям, полученным со спутников Landsat-7/8 и Sentinel-2A/B. Космические изображения загружались из Интернет-архива системы Earth Explorer. По итогам контроля качества и коэффициента покрытия облачностью для обработки было отобрано в общей сложности 109 космических изображений.

Реализация работы предложенного метода осуществлялась путём комбинирования функций программных пакетов SNAP, ENVI, IDL, QGIS, MSExcel, а также встраиваемых в интерфейс ENVI специализированных программных модулей НИИ «Аэрокосмос». На данном этапе исследования обработка выполнялась согласно интерактивному способу (функциональный блок на схеме на рис.1 слева).

Визуализация и анализ результатов обработки космических оптических изображений проводились с помощью геоинформационной системы (ГИС), разработанной в рамках рассматриваемого кейса исследований. Целесообразность использования ГИС при мониторинге морских акваторий показана в ряде работ (например [2,16]). При создании ГИС в качестве базовой информационной основы использовались различные типы пространственных данных, в том числе карты течений, глубин, судоходных трасс, объектов, характеризующих нефтегазоносность [17], и др. Правильно подобранная базовая информационная основа ГИС обеспечивает повышение качества интерпретации регистрируемых по космическим изображениям признаков загрязнений водной поверхности [16].

Согласно схеме, приведённой на рис.1, на заключительном этапе работы метода поиска и идентификации загрязнений водной поверхности по космическим оптическим изображениям выполняется подготовка информационных продуктов в виде тематических карт, инфографики, отчётов. Пример информационного продукта, сформированного по результатам экспериментальной апробации предложенного метода, приведён на рис.4.

В результате мониторинга нефтяных загрязнений в акватории российского сектора Чёрного моря вблизи полуострова Крым с использованием разработанного метода поиска и идентификации загрязнений водной поверхности по 109-и космическим оптическим изображениям было выявлено 92 загрязнённых участка общей площадью 327,63 км² (см. чёрные пятна на рис.4). Наиболее обширный участок характеризовался площадью 99,71 км². Отношение площади всех нефтезагрязнённых участков к площади зоны исследования составило 0,24 \%. 
По итогам анализа БДСХ была выявлена стабильность характеристик спектральных контрастов оптических аномалий, обусловленных нефтяными загрязнениями водной поверхности (для случая, когда нефть визуализируется изображениях в виде пятен с отрицательными контрастами), что можно наблюдать на графиках, приведённых на врезке рис.4 справа внизу. В данном исследовании контрасты вычислялись, как разности между радиометрически-калиброванными средним значениями спектральной отражательной способности нефтезагрязненного и фонового участков водной поверхности. Перед вычислением контрастов осреднённые спектральные кривые фоновой и загрязнённой водной поверхности нормировались на величину отражательной способности в синем канале, что позволило исключить из анализа сильно вариабельную величину интегрального контраста и сосредоточиться на изучении спектральных особенностей регистрируемых оптических аномалий. Отметим, что аналогичные исследования оптических характеристик поверхностных проявлений сточных вод также обнаруживают стабильность спектральных контрастов для такого вида загрязнений [15].

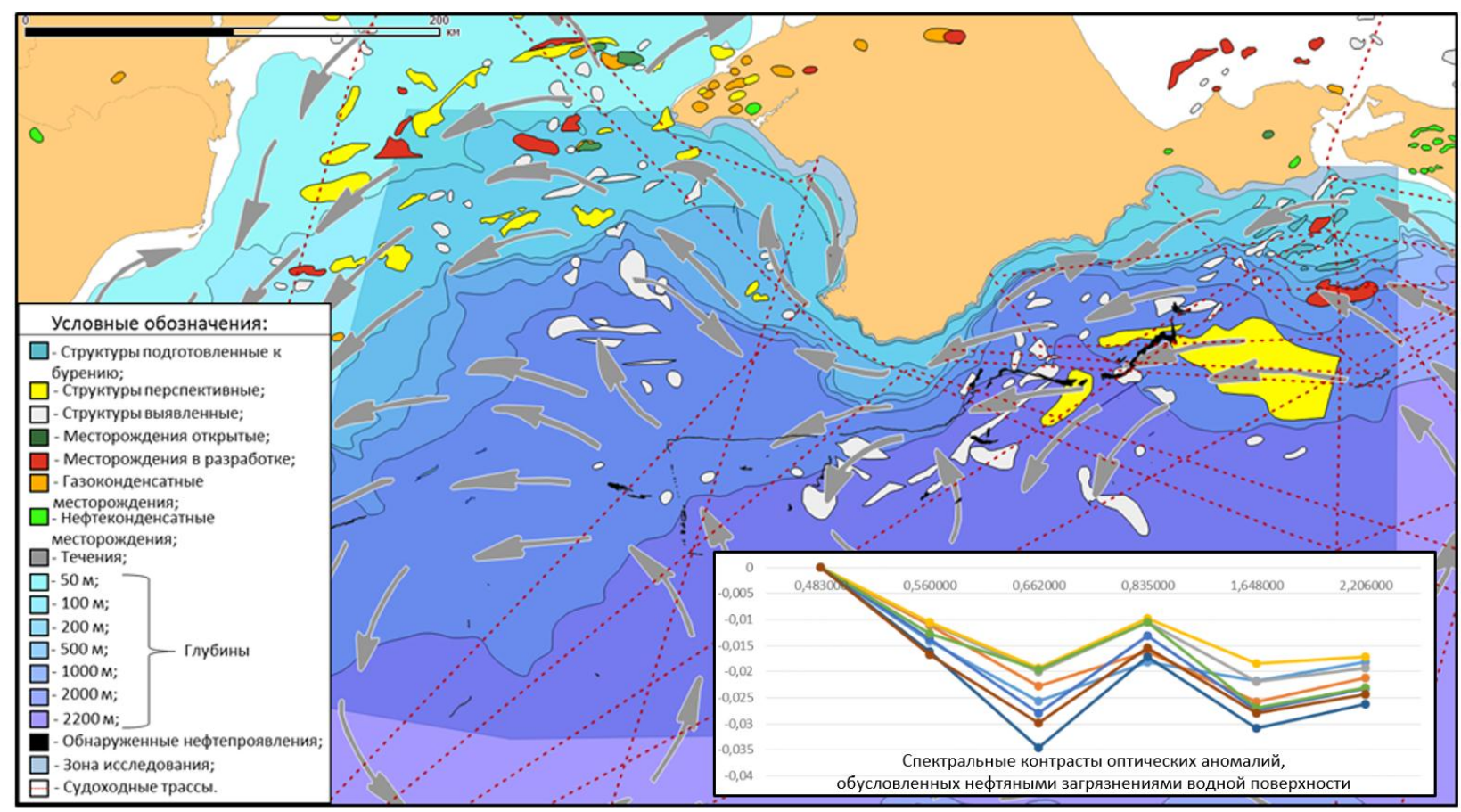

Рис. 3. Пример комплексного информационного продукта, полученного в результате мониторинга

нефтяных загрязнений в акватории российского сектора Чёрного моря вблизи полуострова Крым с использованием разработанного метода поиска и идентификации загрязнений водной поверхности по космическим оптическим изображениям (период мониторинга - с марта 2017 года по август 2018 года,

использованы данные спутников Landsat-7/8, Sentinel-2A/B): ситуационная карта акватории с

результатами обнаружения нефтепроявлений; условные обозначения (слева внизу); спектральные контрасты оптических аномалий, обусловленных нефтяными загрязнениями водной поверхности (справа внизу). Стрелками показан режим течений

\section{Заключение}

Предложен автоматизированный метод поиска и идентификации загрязнений водной поверхности по космическим оптическим изображениям. Метод предполагает постепенное накопление базы данных спектральных характеристик оптических аномалий, обусловленных различными типами загрязнений водной поверхности, и использование такой базы данных для повышения степени автоматизации обработки потоков информации в системах космического мониторинга акваторий морей и океанов. Выявлена стабильность рассчитанных по оригинальной методике характеристик спектральных контрастов оптических аномалий, обусловленных нефтяными загрязнениями водной поверхности, что обнаруживает перспективы для 
продолжения исследований в части автоматизации процессов поиска и идентификации таких аномалий по космическим оптическим многоспектральным изображениям.

В результате мониторинга нефтяных загрязнений в акватории российского сектора Чёрного моря вблизи полуострова Крым с использованием разработанного метода поиска и идентификации загрязнений водной поверхности по космическим оптическим изображениям было выявлено 92 загрязнённых участка общей площадью 327,63 км². Наиболее обширный нефтезагрязнённый участок характеризовался площадью 99,71 км². Отношение площади всех обнаруженных нефтезагрязнённых участков к площади зоны исследования составило 0,24 \%. В общей сложности было обработано 109 космический изображений, полученных с марта 2017 года по август 2018 года со спутников Landsat-7/8 и Sentinel-2A/B.

Работа выполнена при поддержке Минобрнауки России.

\section{References}

[1] Bondur V. Complex Satellite Monitoring of Coastal Water Areas // 31st International Symposium on Remote Sensing of Environment. ISRSE, 2005. 7p.

[2] Bondur V.G. Satellite monitoring and mathematical modelling of deep runoff turbulent jets in coastal water areas // in book Waste Water - Evaluation and Management, ISBN 978-953-307-233-3, InTech, Croatia. 2011. P. 155-180. http:// www.intechopen.com/articles/show/title/satellite-monitoring-and-mathematical-modelling-ofdeep-runoff-turbulent-jets-in-coastal-water-areas

[3] Lavrova O. Yu., Kostianoy A. G., Lebedev S. A., Mityagina M. I., Ginzburg A. I., Sheremet N. A. Kompleksnyy sputnikovyy monitoring morey Rossii (Complex Satellite Monitoring of the Russian Seas). Moscow: IKI Publishing, 2011. 472 p. (In Russian)

[4] Bondur V.G., Keeler R.N., Starchenkov S.A., Rybakova N.I. Monitoring zagryazneniy pribrezhnykh akvatoriy $\mathrm{s}$ ispolzovaniem mnogospektral'nykh sputnikovykh izobrazheniy vysokogo prostranstvennogo razresheniya (Monitoring of the pollution of the ocean coastal water areas using space multispectral high resolution imagery) // Issledovanie Zemli iz kosmosa. 2006. No. 6. P. $42-49$ (In Russian).

[5] Bondur V.G. Aerospace Methods and Technologies for Monitoring Oil and Gas Areas and Facilities // Izvestiya, Atmospheric and Oceanic Physics. 2011. Vol. 47. No. 9. P. 1007-1018. DOI: $10.1134 /$ S0001433811090039

[6] Bondur V.G., Filatov N.N., Grebenyuk Yu.V., Dolotov Yu.S., Zdorovennov R.E., Petrov M.P., Tsidilina M.N. Studies of hydrophysical processes during monitoring of the anthropogenic impact on coastal basins using the example of Mamala Bay of Oahu Island in Hawaii // Oceanology. 2007. Vol. 47. No. 6. P. 769-787. DOI: $10.1134 /$ S0001437007060033

[7] Krovotyntsev V.A., O.YU. Lavrova, M.I. Mityagina, A.G. Ostrovskiy Kosmicheskiy monitoring sostoyaniya prirodnoy sredy Azovo-Chernomorskogo basseyna // Sovremennyye problemy distantsionnogo zondirovaniya zemli iz kosmosa. 2007. T.4. № 1. C. 295-303.

[8] Bondur V.G., Zhurbas V.M., Grebenyuk Yu.V. Mathematical Modeling of Turbulent Jets of Deep-Water Sewage Discharge into Coastal Basins // Oceanology. 2006. Vol.46. No.6. P. 757-771. DOI: $10.1134 / \mathrm{S} 0001437006060014$

[9] Bondur V.G., Grebenyuk Yu.V., Sabinin K.D. Variability of internal tides in the coastal water area of Oahu Island (Hawaii) // Oceanology. 2008. Vol. 48. No. 5. pp.611-621. DOI: 10.1134/S0001437008050019

[10] Bondur V.G., Grebenyuk Yu.V., Sabinin K.D. Variability of internal tides in the coastal water area of Oahu Island (Hawaii) // Oceanology. 2008. Vol. 48. No. 5. pp.611-621. DOI:10.1134/S0001437008050019 
[11] Bondur V, Tsidilina M. Features of Formation of Remote Sensing and Sea truth Databases for The Monitoring of Anthropogenic Impact on Ecosystems of Coastal Water Areas // 31st International Symposium on Remote Sensing of Environment. ISRSE, 2005. P. 192-195.

[12] Bondur V.G., Grebenuk Y.V. Distantsionnaya indikatsiya antropogennykh vozdeystviy na morskuyu sredu, vyzvannykh zaglublennymi stokami: modelirovanie, eksperimenty (Remote indication of anthropogenic influence on marine environment caused by depth wastewater plume: Modelling, experiments) // Issledovanie Zemli iz Kosmosa. 2001. No. 6. P. 49-67. (In Russian)

[13] Bondur V.G., Zubkov E.V. Vydelenie melkomasshtabnykh neodnorodnostey opticheskikh kharakteristik verkhnego sloya okeana po mnogozonalnym sputnikovym izobrazheniyam vysokogo razresheniya. Chast 1. Effekty sbrosa drenazhnykh kanalov v pribrezhnye akvatorii (Showing up the small-scale ocean upper layer optical inhomogeneities by the multispectral space images with the high surface resolution. Part 1 . The canals and channels drainage effects at the coastal zone) // Issledovaniya Zemli iz kosmosa. 2005. No. 4. P. 54-61. (In Russian)

[14] Akovetskiy V.G. Aerokosmicheskiy monitoring mestorozhdeniy nefti i gaza (Aerospace monitoring of oil and gas deposits). Moscow: Nedra-Businnes Centre, 2008. 454 p. (In Russian)

[15] Bondur V.G., Vorobyev V.E., Zamshin V.V., Serebryany A.N., Latushkin A.A., Li M.E., Martynov O.V., Hurchak A.P., Grinchenko D.V. Monitoring anthropogenic impact on some coastal water areas of the Black Sea using multispectral satellite imagery // Izvestiya, Atmospheric and Oceanic Physics, 2018, Vol. 54, No. 9, pp. 1008-1022. DOI: 10.1134/S0001433818090098.

[16] Ivanov A.Yu., Zatyagalova V.V. A GIS approach to mapping of oil spills in the marine environment // International Journal of Remote Sensing, 2008. V. 29. N 21. P. 6297-6313.

[17] Glumov I.F., Gulev V.L., Senin B.V., Karnaukhov S.M. Regionalnaya geologiya i perspektivy neftegazonosnosti CHernomorskoy glubokovodnoy vpadiny i prilegayushchikh shelfovykh zon (Regional geology and the prospects of hydrocarbon potential of the Black Sea trench and adjacent shelf zones) / Ed. by B.V. Senin. In 2 parts. Part 1. Moscow: Nedra, 2014. (In Russian) 